CHAPTER?

\title{
Development of Methodology to Gather Seated Anthropometry Data in a Microgravity Environment
}

\author{
Karen Young, Miranda Mesloh, Sudhakar Rajulu \\ Anthropometry and Biomechanics Facility \\ NASA, Johnson Space Center \\ Houston, TX 77058, USA
}

\begin{abstract}
The Constellation Program is designing a new vehicle based off of new anthropometric requirements. These requirements specify the need to account for a spinal elongation factor for anthropometric measurements involving the spine, such as eye height and seated height. However, to date there is no data relating spinal elongation to a seated posture. Only data relating spinal elongation to stature has been collected in microgravity. Therefore, it was proposed to collect seated height in microgravity to provide the Constellation designers appropriate data for their analyses. This document will describe the process in which the best method to collect seated height in microgravity was developed.
\end{abstract}


Keywords: Microgravity, Spinal Elongation, Human System Integration, NASA

\section{INTRODUCTION}

The Constellation Program's Crew Exploration Vehicle (CEV) is required to accommodate the full anthropometric range of the future crewmember population according to the requirements stated in the Human-Systems Integration Requirement (HSIR) document (CxP70024). One critical anthropometric measurement for the CEV is seated height. Seated height allows for the CEV designers to determine the optimum seat configuration in the vehicle. Changes in seated height can have a large impact on the design, on accommodation, and safety of the crewmembers. Historically it is known that the spine changes in microgravity due to spinal elongation which will affect crewmembers' seated height.

Spinal elongation is the straightening of the natural curvature of the spine and the expansion of intervertebral disks. This straightening results from fluid shifts in the body and the lack of compressive forces on the spinal vertebrae in microgravity. Previous studies have shown that as the natural curvature of the spine straightens, an increase in overall height of $3 \%$ of stature occurs, and is the basis of the current HSIR requirements (NASA Ref. 1024). However, because of variations in the torso/leg ratio, questions arose regarding if the historical stature data can be applied to seated measurements. Data related to how spinal elongation specifically affects seated measurements is nonexistent.

An experiment was designed to collect spinal elongation data while subjects were in a seated posture in microgravity. The purpose of this study was to provide quantitative data representing the amount of change that occurs in seated height due to spinal elongation in microgravity environments.

While preparing for the payload experiment concerns arose regarding the possibility of crewmembers not restraining themselves properly to the seat pan. In order to resolve this concern and ensure that the measurements of seated height were accurately collected during the experiment, a simulated microgravity experiment was conducted. A simulated microgravity condition (parabolic flight) was used to conduct three evaluations to test the methodology and procedures of the experiment. During these simulated evaluations data was collected to a) ensure that the lap restraint method provided sufficient restraint to eliminate any gap between the subject's gluteal surface and the seat pan and b) document any necessary design and procedural changes needed because of the microgravity environment.

\section{METHOD}

One of the major concerns regarding the data collection of seated height in microgravity was the potential separation between the subject and the seat pan. While the crewmember is expected to wear a lap restraint, it was unclear whether the lap restraint's tension, set by the individual crewmember, would be able to 


\section{[Type text]}

restrain the individual to allow for accurate data collection. Three simulated microgravity flights occurred during which seated height, force data, and pressure data was collected.

The test setup included the Shuttle seat and an anthropometer. For Flight 1 and Flight 2, a standard anthropometer was attached to the Shuttle seat, and during Flight 3 a prototype of the flight hardware anthropometer was used which attached to the top of the seat back in the same manner as the head rest. During the simulated micro-gravity parabolas, the subject was seated in the Shuttle seat and restrained for the collection of pressure, force, and seated height data. The subject remained seated during several consecutive parabolas; the number of consecutive parabolas varied between subjects.

Flight 1 was performed to determine if the current hardware on the Shuttle seat was able to ensure proper contact with the seat pan while not affecting the seated height measurements. This was the proposed method of restraint for the payload experiment. The restraint system examined was 3-points of the current 5point harness on the Shuttle seat. The 3-point harness included the use of the crotch buckle and the two pelvic straps. Data was collected for four subjects using this restraint method.

After learning the results of the first flight, alternative restraint methods were proposed and examined during the second flight. The restraint systems examined were iterations of the restraint method tested during the first flight. However, in discussing the concerns with the stakeholder an additional restraint method was tested during the flight, adjusting the $3 \mathrm{pt}$ harness by wrapping it around the seatback support of the seat. Four subjects participated in Flight 2 in which pressure data and seated height data was collected.

The third flight, Flight 3 , tested the prototype anthropometer assembly and the optimized restraint method from Flight 2, the 3pt harness wrapped around the seatback support bars. Seated height and pressure data was collected from three subjects during Flight 3.

Seated height data was collected during all three flights for each restraint system examined. The seated height data was used to validate which restraint system secured the subject to the seat pan with the smallest amount of variability between the measurements. To ensure minimal variability to the measure, all measurers followed a standard procedure. Once in microgravity, the measurer ensured the subject was looking straight and collected seated height measurement ensuring proper placement and proper contact on the top of the subject's head. For Flights 1 and 2, one seated height measurement was recorded per parabola. During Flight 3, multiple seated height measurements were recorded per parabola due to time allotment and procedure familiarization of the measurers. 


\section{RESULTS}

\section{FLIGHT 1}

Seated height measurements were collected during consecutive parabolas for each subject. The total number of data points differed for each subject. The restraint method used during Flight 1 was the 3-point harness of the current hardware on the Shuttle seat. The results showed that the range of seated height measurements were greater than $1 \mathrm{~cm}$ and varied from $1.1 \mathrm{~cm}$ to $5.6 \mathrm{~cm}$ (Table 1$)$. The variability in the seated height measurements indicate that the subjects were not tightly restrained and that the subjects buttocks were not in complete contact with the seat pan. The subjects also reported the lack of restraint; the subjects often felt like their buttocks was floating off of the seat in micro-gravity, even with the lap restraint tightened as far as the restraint system would allow.

Table 1 shows that subject 3 had the most variability in seated height which may indicate that this subject was not consistently in contact with the seat pan for all trials. Subject 4 experienced the smallest range of values during microgravity. The smaller the range of seated height measurements, the higher the repeatability of the measurement and the repeatability of tightening the restraint system in the same manner for multiple parabolas.

The results from Flight 1 demonstrate that the current 3-point harness used in its nominal configuration was not sufficient to ensure contact between the subject and the seat pan to collect consistent seated height measurements. The restraint system must restrain the subject properly so that the seated height measurements are consistent. Inconsistent measurements will adversely affect the amount of spinal growth that is reported to the designers, which may impact crew safety and crew fit with the seats, suits, and vehicle after exposure to microgravity.

Table 1. Flight 1 (F1) Seated Height Measurements
\begin{tabular}{|c|c|c|c|c|} 
& \multicolumn{4}{|c|}{ 3-point Nominal Harness (cm) } \\
\hline & Subject 1 & Subject 2 & Subject 3 & Subject 4 \\
\hline Range & 1.4 & 2.3 & 5.6 & 1.1 \\
\hline Mean & 95.4 & 90.3 & 90.5 & 87.1 \\
\hline
\end{tabular}

\section{FLIGHT 2}

As a result of Flight 1, that the 3-point harness in its nominal configuration was not sufficient to restrain the subject, alternative methods of restraints were explored during Flight 2, namely:

- Flight 13 pt configuration

- Flight $13 \mathrm{pt}$ configuration with a foam insert under the buckle

- A 3pt harness with a loose buckle and a Velcro leg strap

- Flight $23 \mathrm{pt}$ harness configuration

- Flight 2 3pt configuration with Velcro leg strap 


\section{[Type text]}

- Flight $23 p t$ configuration with Velcro leg and lap straps

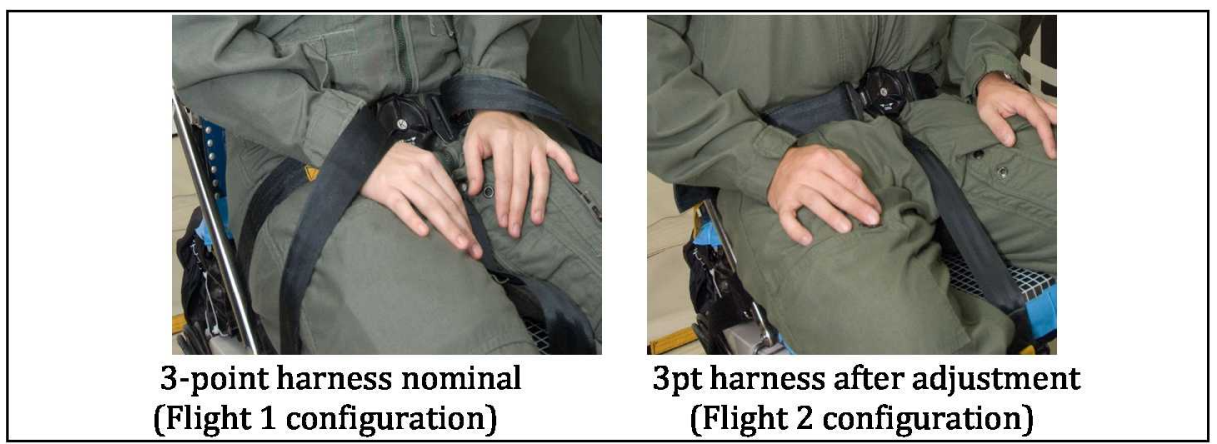

Figure 1: Harness Configurations

In Figure 1 above, Flight 1 configuration represents the attachment point location of the waist belts of the $3 \mathrm{pt}$ harness. Flight 1 configuration consists of the waist belts being attached and used in the normal configuration. Whereas, Flight 2 configuration consists of the waist belts being wrapped around the seatback support bars and then attached to the buckle. The Flight 2 configuration brought the buckle up higher and tighter on the pelvic region. The Flight 2 configuration also pulled the subject back and down into the seat whereas the Flight 1 configuration only pulled the subject down into the seat and leaving room for the subjects' buttock to rise off of the seat.

According to the seated height measurements collected during Flight 2, the seated height range verified that the Flight 2 configuration restrained the subject(s) more efficiently than the Flight 1 configuration. The greatest range in seated height for subject 1 was $0.5 \mathrm{~cm}$ for Flight 2 (3pt w/ leg \& lap configuration) compared to $1.4 \mathrm{~cm}$ for Flight 1 (Table $1 \& 2$ ). This indicates that the subject was better restrained in the seat for Flight 2 than for Flight 1. Looking at the three different types of restraints and the ranges of measurement variation, the data indicates that the addition of the leg and lap straps minimize the seated height range, except for subject 1 . This would signify that the addition of a leg and lap strap may be necessary to restrain the subject to the seat and allow for accurate seated height measurements. However, when comparing the average seated height measurements for each configuration, the seated height measurements did not vary greatly between the different configurations (Table 3). Therefore, to reduce the need for extra hardware in space, the Flight 2 3-point harness was chosen as the best restraint configuration. The differences in the average seated heights can be accounted for the variation that occurs while collecting anthropometric measurements using traditional methods, i.e. manual measurements using an anthropometer $(0.6 \mathrm{~cm}$ for seated height). 
Table 2. Flight 2 (F2) Seated Height Measurements

\begin{tabular}{|c|c|c|c|c|c|c|c|c|}
\hline \multicolumn{8}{|c|}{ Flight 2 } \\
\hline & \multicolumn{7}{|c|}{ 3-point (cm) } & \multicolumn{3}{c|}{$\begin{array}{c}\text { 3-point w/ leg strap } \\
\text { (cm) }\end{array}$} & $\begin{array}{c}\text { 3-point w/ leg \& } \\
\text { lap strap (cm) }\end{array}$ \\
\hline & S 1 & S 2 & S 5 & S 1 & S 2 & S 5 & S 1 & S 2 \\
\hline Range & 0.1 & 0.3 & 1.2 & 0.4 & 0.1 & 0.1 & 0.5 & 0.1 \\
\hline Mean & 94.0 & 90.0 & 94.2 & 93.7 & 90.1 & 94.1 & 93.6 & 89.9 \\
\hline
\end{tabular}

Table 3. Average Seated Height Configuration Comparison

\begin{tabular}{|l|c|c|c|}
\hline \multicolumn{1}{|c|}{ Restraint Configuration } & S1 (cm) & S 2 (cm) & S5 (cm) \\
\hline Flight 2 3pt & 94.0 & 90.0 & 94.2 \\
\hline Flight 2 3pt w/ Leg Strap & 93.7 & 90.1 & 94.1 \\
\hline Flight 2 3pt w/ Leg \& Lap Straps & 93.6 & 89.9 & - \\
\hline Flight 1 3pt & 95.4 & 90.2 & - \\
\hline
\end{tabular}

\section{FLIGHT 3}

Flight 3 tested the Flight $23 \mathrm{pt}$ harness configuration in addition to the use of the prototype anthropometer to be used during the payload experiment. The prototype anthropometer attaches to the seatback in the same manner as the headrest. In addition to testing the 3-point harness, the subjects were also asked to look up, down, and straight forward to determine if the head position does affect the seated height. During Flight 3, it was also apparent that the operator and subject must work together to ensure that the restraint system is tight against the subject's pelvis. Several times the restraint was not completely tight, and the subjects responded by stating that they were not synched down completely and felt as if they were floating. The mean and range of seated height measurements for the different condition, head orientations and restraint tightness, can be seen in Table 4.

Table 4. Flight 3 Seated Height Measurements

\begin{tabular}{|c|c|c|c|c|c|c|c|c|c|c|}
\hline \multicolumn{11}{|c|}{ Flight 3} \\
\hline & \multicolumn{3}{|c|}{$\begin{array}{l}\text { Restraint Loose, } \\
\text { Looking Forward } \\
\text { (cm) }\end{array}$} & \multicolumn{3}{|c|}{$\begin{array}{c}\text { Restraint Tight, } \\
\text { Looking Forward } \\
\text { (cm) }\end{array}$} & \multicolumn{2}{|c|}{$\begin{array}{c}\text { Looking Up } \\
(\mathbf{c m})\end{array}$} & \multicolumn{2}{|c|}{$\begin{array}{l}\text { Looking } \\
\text { Down }(\mathrm{cm})\end{array}$} \\
\hline & S 1 & S 2 & S 3 & s 1 & S 2 & S 3 & S 1 & S 2 & S 1 & S 2 \\
\hline Range & 0.9 & 1.4 & 0.1 & 0.6 & 0.6 & 0.7 & 0.5 & 0 & 0.6 & 0 \\
\hline Mean & 95.3 & 91.4 & 94.7 & 95.4 & 91.1 & 93.7 & 95.2 & 90.7 & 93.3 & 91.2 \\
\hline
\end{tabular}

When the subjects were loosely restrained versus tightly restrained with the $3 \mathrm{pt}$ harness, the range of the seated height measurements decreased. Subject 2's measurement range decreased from $1.4 \mathrm{~cm}$ to $0.6 \mathrm{~cm}$ due to tightening of the restraint system (Table 4). All three subjects seated height ranges were similar when they were tightly restrained, $0.6 \mathrm{~cm}, 0.6 \mathrm{~cm}$, and $0.7 \mathrm{~cm}$, indicating that when tightly restrained the variation in seated height is due to the effects of microgravity and the variation in collecting manual measurements (as previously described) (Table 4). 


\section{[Type text]}

Based on the limited amount of data collected during Flight 3 in which the subjects changed their head orientation from looking forward, down, and up, the seated height measurements did change for subject 1 and subject 2 . When comparing looking forward to looking down, there was a greater increase in seated height for subject 1 compared to subject 2 . This resulted from subject 2 drastically looked down as opposed to subject 2 who did not drastically look down at the floor. This drastic change in head orientation was not seen when the subjects looked up. Therefore, the difference in seated heights when the subjects looked up versus looking forward was very small, $0.2 \mathrm{~cm}$ and $0.4 \mathrm{~cm}$ respectively for subjects 1 and 2. Another factor that may explain the small variation in seated height values when looking up was the location on the head where the measurement was collected. The measurements may not have been collected from the most superior location on the head but rather to the middle of the head. From the results of looking down, it can be assumed that if the subjects drastically looked up and the measurement was collected at the most superior location on the head then the seated height measurements would be affected the same as when the subjects drastically looked downward. If the seated height of the payload experiment were to be recorded when the subjects were not looking forward then the results from the study that would be given to the designers and used to update HSIR requirements would be overcalculated, impacting crew safety, crew selection, and anthropometric requirements.

\section{DISCUSSION}

\section{FLIGHT 1}

The results obtained from Flight 1 were: 1) the current 3 pt harness did not ensure complete contact between the subject and the seat pan and 2) the set-up, harness and anthropometer, affected the seated height measurements consistency. The subjects reported verbally that they often felt as if they were not completely restrained and experienced a sensation of floating between the seat pan and their gluteal surface. These results may be due to the configuration of the restraint system. The configuration of the $3 \mathrm{pt}$ restraint system for Flight 1 rested lower on the pelvic region. The crotch buckle was not adjusted by each subject nor was its length extended completely, resulting in the buckle attachment point resting lower on the pelvic region that allowed for a gap to exist between the buckle and the subject. This gap allowed the subject to float unrestrained until the subject was stopped by the restraint buckle (Figure 1).

Two lessons learned while collecting seated height measurements during Flight 1 were: 1) to make sure the subject is looking straight forward, and 2) make sure the subject is in the center of the seat. Oftentimes, the subject perceived that they were looking forward but they were actually looking slightly downwards. Also, there were times when the anthropometer was not positioned in the middle of the subject's head due to their positioning on the chair. Therefore, the measurer needs to instruct the subject as to how to move their torso/head so that the anthropometer measures to the middle of the top of the head. The two lessons learned can 
adversely affect the measurement of seated height by providing inaccurate data. Without accurate data, the impact of spinal elongation on seat positioning and fit may pose risks to crew safety.

\section{FLIGHT 2}

The findings from Flight 2 indicated: 1) additional straps across the waist and lower thighs decrease the range in seated height measurements, and 2) that the waist restraint should be rerouted through the back of the seatback, this increased the consistency in measurements and the contact between the subject and the seat. The subjects felt greater restrained with this configuration than the configuration of Flight 1 (Figure 1).

The average seated heights results from this flight demonstrated that additional straps were not necessary. Therefore, additional hardware was not needed for the on-orbit activity. The decision was made to proceed with the Flight $23 \mathrm{pt}$ harness configuration for on-orbit activities; wrapping the restraint belts around the seatback joint.

\section{FLIGHT 3}

Flight 3 only tested the Flight $23 \mathrm{pt}$ harness configuration utilizing the prototype hardware anthropometer. The prototype hardware attached to the seat in the same manner as the headrest. Using the prototype anthropometer during Flight 3, resulted in little variability during parabolas and decreased the inconsistencies of using a standard anthropometer taped to the load cell plate. Also, using the Flight $23 \mathrm{pt}$ harness configuration decreased the range for seated height due to the tightening of the restraint. During Flight 3, several seated height measurements were collected when the restraint was not completely tight, resulting in a greater range than when the restraint was pulled tight around the subject's pelvic/waist area (Table 5). The lack of tightness led to inconsistencies within the seated height measurements. As a result, a lesson learned from Flight 3 is that after the subject gets in the seat and is buckled the operator must pull the side straps as tight as he/she can to ensure the subject is in contact with the seat pan. 


\section{[Type text]}

Table 5. Flight 3 Seated Height Measurements Based on Restraint Tightness and Head Position

\begin{tabular}{|c|c|c|c|c|c|c|c|c|}
\hline & \multicolumn{2}{|c|}{$\begin{array}{c}\text { Straps Loose - } \\
\text { Looking Straight }\end{array}$} & \multicolumn{2}{c|}{$\begin{array}{c}\text { Straps Tight - } \\
\text { Looking Straight }\end{array}$} & \multicolumn{2}{c|}{$\begin{array}{c}\text { Straps Tight - } \\
\text { Looking Up }\end{array}$} & \multicolumn{2}{c|}{$\begin{array}{c}\text { Straps Tight - } \\
\text { Looking Down }\end{array}$} \\
\hline Subject & $\begin{array}{c}\text { Mean } \\
(\mathbf{c m})\end{array}$ & $\begin{array}{c}\text { Zero-g } \\
\text { Range } \\
(\mathbf{c m})\end{array}$ & $\begin{array}{c}\text { Mean } \\
(\mathbf{c m})\end{array}$ & $\begin{array}{c}\text { Zero-g } \\
\text { Range } \\
\text { (cm) }\end{array}$ & $\begin{array}{c}\text { Mean } \\
(\mathbf{c m})\end{array}$ & $\begin{array}{c}\text { Zero-g } \\
\text { Range } \\
(\mathbf{c m})\end{array}$ & $\begin{array}{c}\text { Mean } \\
\text { (cm) }\end{array}$ & $\begin{array}{c}\text { Zero-g } \\
\text { Range } \\
(\mathbf{c m})\end{array}$ \\
\hline S1 & 95.3 & 0.9 & 95.4 & 0.6 & 95.2 & 0.5 & 93.3 & 0.6 \\
\hline S2 & 91.4 & 1.4 & 91.1 & 0.6 & $90.7^{*}$ & - & $91.2^{*}$ & - \\
\hline S3 & 94.7 & 0.1 & 93.7 & 0.7 & - & - & - & - \\
\hline \multicolumn{7}{|c|}{ *easurement was taken with loose straps }
\end{tabular}

Comparing the average seated height for the $3 \mathrm{pt}$ harness restraint system for all three flights, it was determined that the Flight 3 configuration improved the variability compared to Flight 1 (Table 6). Flight 2 had the smallest seated height range but had the fewest seated height measurements for each subject; subject 1 had two measurements and subject 2 had three measurements.

Table 6. Comparison of Seated Height 3pt Harness Configuration

\begin{tabular}{|l|c|c|c|c|c|c|}
\hline & \multicolumn{2}{|c|}{ Subject 1 } & \multicolumn{2}{c|}{ Subject 2 } & \multicolumn{2}{c|}{ Subject 3 } \\
\hline & $\begin{array}{c}\text { Average } \\
\text { (cm) }\end{array}$ & $\begin{array}{c}\text { Range } \\
(\mathrm{cm})\end{array}$ & $\begin{array}{c}\text { Average } \\
(\mathrm{cm})\end{array}$ & $\begin{array}{c}\text { Range } \\
(\mathrm{cm})\end{array}$ & $\begin{array}{c}\text { Average } \\
(\mathrm{cm})\end{array}$ & $\begin{array}{c}\text { Range } \\
(\mathrm{cm})\end{array}$ \\
\hline Flight 1 & 95.4 & 1.4 & 90.3 & 2.3 & 90.5 & 5.6 \\
\hline Flight 2 & 94.0 & 0.1 & 90.0 & 0.3 & - & - \\
\hline Flight 3 & 95.4 & 0.6 & 91.1 & 0.6 & 93.7 & 0.7 \\
\hline
\end{tabular}

\section{CONCLUSION}

In conclusion, the microgravity flights were pertinent to the Spinal Elongation payload experiment. Flight 1 verified that the current $3 \mathrm{pt}$ harness system was not sufficient to provide adequate contact between the seat pan and the subject. Flight 1 also provided insight into some possible procedural problems that may occur. The lessons learned during Flight 1 were: 1 ) the subjects often perceived that they were looking straight when they were looking slightly downward, and 2) to confirm that the subjects were in the center of the chair so that the anthropometer was measuring to the middle of their head. These lessons learned have been included into the crew procedures, ensuring that crewmembers are aware these issues and can position the subjects as necessary to achieve an accurate seated height measurement.

After learning that the current restraint system was not sufficient seven alternative restraint systems were examined during Flight 2 . The results from Flight 2 indicated that a restraint on the lower thighs and a restraint that is anchored from behind the seatback will pull the subject down and back into the seat thereby reducing the variability in the seated height measurement. However, the seated height data from Flight 2 proved that the variability between the Flight $23 \mathrm{pt}$ harness, the $3 \mathrm{pt}$ harness with a leg strap, and the $3 \mathrm{pt}$ harness with the leg and lap 
straps was minor. Therefore, due to time and schedule constraints, the decision was made by the Principal Investigative team not to further develop any additional hardware.

With the restraint system decided upon, Flight 3 examined the design of the prototype anthropometer and operationally verified the restraint method for crew procedures. One lesson learned during Flight 3 was that the lap straps of the restraint must be tight against the subjects' pelvis/waist to reduce the amount of variability in the seated height measurements.

The three microgravity flights allowed the Principal Investigation team to explore the best methodology for collecting seated height data. The seated height data collected during the microgravity flights assisted with crew procedures, data collection methodology, and hardware design. The lessons learned from the microgravity flights will aide in successfully and accurately collecting seated height data that can be used by the CEV designers to accurately design for the amount spinal growth due to microgravity. The spinal growth that crewmembers may experience will effect crew safety, crew selection, and design requirements. If the designers do not have the correct data for spinal growth, then a crewmember may not have enough clearance upon re-entry to allow for the appropriate vibration and stroke volume or may not properly fit into their re-entry suit therefore affecting their safety and the safety of the other crewmembers.

\section{REFERENCES}

Anthropometric Source Book Vol. I: Anthropometry for Designers., (NASA 1024). Edited by Staff of Anthropology Research Project, Webb Associates, Yellow Springs, Ohio, 1978

NASA-STD-3000 (1985, Rev B). Man-Systems Integration Standards, Johnson Space Center, Houston, Texas.

CxP 70024, Human-Systems Integration Requirements (HSIR) (C000114), NASA Johnson Space Center, Houston, TX, 2007 